\title{
Hopping on the Bethe lattice: Exact results for densities of states and dynamical mean-field theory
}

\author{
Martin Eckstein, ${ }^{1}$ Marcus Kollar, ${ }^{1}$ Krzysztof Byczuk, ${ }^{2}$ and Dieter Vollhardt ${ }^{1}$ \\ ${ }^{1}$ Theoretical Physics III, Center for Electronic Correlations and Magnetism, Institute for Physics, University of Augsburg, \\ D-86135 Augsburg, Germany \\ ${ }^{2}$ Institute of Theoretical Physics, Warsaw University, ul. Hoża 69, PL-00-681 Warszawa, Poland \\ (Received 29 September 2004; revised manuscript received 10 January 2005; published 30 June 2005)
}

\begin{abstract}
We derive an operator identity which relates tight-binding Hamiltonians with arbitrary hopping on the Bethe lattice to the Hamiltonian with nearest-neighbor hopping. This provides an exact expression for the density of states (DOS) of a noninteracting quantum-mechanical particle for any hopping. We present analytic results for the DOS corresponding to hopping between nearest and next-nearest neighbors, and also for exponentially decreasing hopping amplitudes. Conversely it is possible to construct a hopping Hamiltonian on the Bethe lattice for any given DOS. These methods are based only on the so-called distance regularity of the infinite Bethe lattice, and not on the absence of loops. Results are also obtained for the triangular Husimi cactus, a recursive lattice with loops. Furthermore we derive the exact self-consistency equations arising in the context of dynamical mean-field theory, which serve as a starting point for studies of Hubbard-type models with frustration.
\end{abstract}

DOI: 10.1103/PhysRevB.71.235119

PACS number(s): 71.10.Fd, 71.27.+a, 05.50.+q, 02.10.Ox

\section{INTRODUCTION}

The Bethe lattice is an infinite graph, where any two points are connected by a single path and each vertex has the same number of branches $Z$, as shown in Fig. 1 for $Z=4$. The name "Bethe lattice" originates from the fact that Bethe's approximation for the Ising model is exact on this lattice. ${ }^{1,2}$ A finite portion of the Bethe lattice is called Cayley tree. The latter has a peculiar thermodynamic limit due to its large surface, ${ }^{2-5}$ whereas the infinite Bethe lattice has no surface, all its lattice sites being located inside the infinite tree.

Strictly speaking the Bethe lattice is a pseudolattice because it does not possess the usual point and translational symmetries of crystal (Bravais) lattices. Nevertheless, it plays an important role in statistical and condensed-matter physics because some problems involving disorder and/or interactions can be solved exactly when defined on a Bethe lattice, e.g., Ising models, ${ }^{1,2,6}$ percolation, ${ }^{7-9}$ or Anderson localization. ${ }^{10-13}$ Such exact solutions on the Bethe lattice for $Z<\infty$ sometimes, ${ }^{1,2}$ but not always, ${ }^{10-13}$ have mean-field character. Furthermore, it was argued that mean-field theories are more reliable if derived on a Bethe lattice. ${ }^{14}$ On the other hand, the Bethe lattice may actually serve as a model for the electronic structure of amorphous solids, as proposed by Weaire and Thorpe,$^{15}$ e.g., for hydrogenated amorphous silicon. ${ }^{16}$ Laughlin and Joannopoulos ${ }^{17}$ used the Bethe lattice to describe lattice vibrations in amorphous silica, and recently this approach was also applied to phonon transport through silica-coated nanowires. ${ }^{18}$

There are two special properties that make the Bethe lattice particularly suited for theoretical investigations. One is its self-similar structure which may lead to recursive solutions. The other is the absence of closed loops which restricts interference effects of quantum-mechanical particles in the case of nearest-neighbor (NN) coupling. The situation is different if also longer-range hopping processes or interactions are allowed, e.g., between next-nearest neighbors (NNN). For example, the frustration introduced by NNN hopping typically suppresses antiferromagnetism in the half-filled Hubbard model at weak coupling.

In this paper we consider tight-binding Hamiltonians describing hopping of a single quantum particle, paying special attention the case where the hopping has both $\mathrm{NN}$ and $\mathrm{NNN}$ contributions (with respective amplitudes $t_{1}, t_{2}$ ), and to the

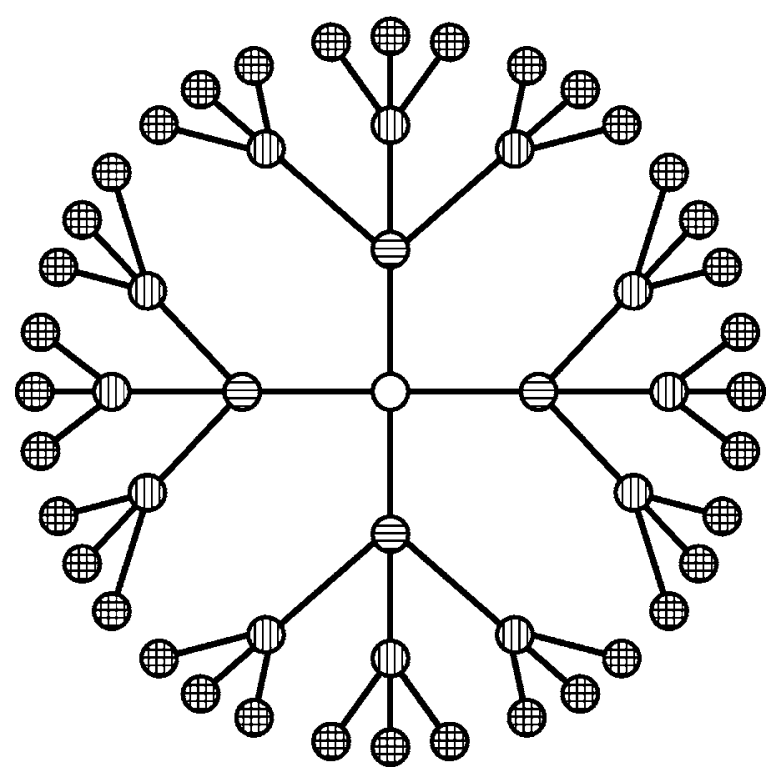

FIG. 1. Part of the Bethe lattice with coordination number $Z=4$. Any two sites are connected by a unique shortest path of bonds. Starting from the site marked by the open circle, horizontally shaded circles can be reached by one lattice step (NN), vertically shaded circles by two lattice steps (NNN), and doubly shaded circles by three lattice steps. Note that the lattice is infinite and that all sites are equivalent; the shading appears only for visualization of hopping processes. 
limit $Z \rightarrow \infty$. The derivation of the spectrum for $\mathrm{NN}$ hopping has a long history involving many different methods. ${ }^{19-23}$ However, these methods are not immediately useful for longer-range hopping. Here we develop a method, based on the algebraic properties of so-called distance-regular graphs, which can effectively treat arbitrary-range hopping on the Bethe lattice and certain other lattices. In particular, we derive an operator identity [Eq. (9)] relating tight-binding Hamiltonians $H_{d}$, which describe hopping between sites that are $d \mathrm{NN}$ steps apart, to $H_{1}$, the Hamiltonian for NN hopping. This general result has several applications. For comparison with earlier methods we first note that the nonlocal Green function for NN hopping can be obtained via a rather short route. We proceed to derive the exact density of states (DOS) for arbitrary hopping and discuss in detail the case of $t_{1}-t_{2}$ hopping and exponentially decreasing hopping. The inverse problem, i.e., the construction of a tight-binding Hamiltonian on the Bethe lattice corresponding to a given DOS, is also solved.

An important limit for any lattice is that of infinite coordination number, $Z \rightarrow \infty$, since it always leads to a mean-field theory of some sort. ${ }^{2}$ It is well known that for fermionic lattice models the hopping matrix elements must then be properly rescaled, e.g., $t_{1} \sim 1 / \sqrt{Z}$ for NN hopping. ${ }^{24}$ In this limit dynamical mean-field theory (DMFT) ${ }^{25-30}$ becomes exact, yielding self-energies that are local in space. In particular the Hubbard model may be mapped onto a single-impurity problem with self-consistency condition. ${ }^{29}$ Here the Bethe lattice leads to further simplifications, e.g., in the solution of the self-consistency equations, partly due to the resulting semielliptical density of states for NN hopping, $\rho(\epsilon)=2 \sqrt{1-(\epsilon / D)^{2}} /(\pi D)$, where $D$ is the halfbandwidth. ${ }^{21,22}$ Interestingly the algebraic band edges of this model DOS resemble those of three-dimensional systems. DMFT with this DOS has been instrumental in clarifying the phase diagram of the Hubbard model, in particular concerning the Mott transition from a paramagnetic metal to a paramagnetic insulator at half-filling. ${ }^{25-35}$

It should be noted that the Mott transition in the Hubbard model with NN hopping is usually hidden by an antiferromagnetic low-temperature phase, ${ }^{26,29,36}$ whereas for transition metal oxides such as $\mathrm{V}_{2} \mathrm{O}_{3}$ the Mott transition line extends beyond the antiferromagnetic phase boundary. ${ }^{37}$ In order to describe this experimental phase diagram at least qualitatively in terms of a Hubbard model, the strong tendency toward antiferromagnetism must be reduced, e.g., by including the ever-present NNN hopping $t_{2}$. This was demonstrated within a DMFT setup in which the DOS remains semielliptic, leading to a suppression of antiferromagnetism without modification of the paramagnetic phase, $;^{29,36,38,39}$ the results of this setup are valid for random hopping on the Bethe lattice. ${ }^{29,36}$ In the present work we consider standard tight-binding hopping without randomness; we find in particular that for $t_{1}-t_{2}$ hopping on the Bethe lattice the DOS is no longer semielliptic but becomes asymmetric. We also evaluate the exact DMFT self-consistency equations for arbitrary hopping on the Bethe lattice, including phases with broken sublattice symmetry. Our results for $t_{1}-t_{2}$ hopping [Eq. (65)] differ from the corresponding equations employed in Refs. 29, 36, 38, and 39; therefore the latter only apply to random hopping.
The paper is structured as follows: In Sec. II we consider topological aspects of tight-binding Hamiltonians. In Sec. III a general operator identity for the Bethe lattice is derived, which is used in Sec. IV to obtain the DOS for various hopping ranges; the Appendix contains similar results for the triangular Husimi cactus. Hopping amplitudes for a given DOS are constructed in Sec. IV D. In Sec. V the DMFT self-consistency equations are derived. Our results are discussed in Sec. VI. Throughout the paper we make contact with particular results for the DOS, Green functions, and DMFT self-consistency equations which were previously obtained by other methods; ${ }^{35,40,41}$ a detailed comparison will be discussed in a separate publication. ${ }^{42}$

\section{TIGHT-BINDING HAMILTONIANS AND TOPOLOGICAL LATTICE PROPERTIES}

In this section we discuss relations among tight-binding Hamiltonians for lattices that belong to a certain class of graphs. In general a graph $G$ is defined as a set of vertices $V=\{i, j, k, \ldots\}$ representing nodes or sites of a network, and a set of links between these vertices. In particular, any Bravais lattice is a graph with vertices and links corresponding to lattice sites and bonds between nearest neighbors, respectively. For a given lattice one distinguishes between the metric and topological distance between two vertices $i$ and $j$. The metric distance is determined by the metric properties of the space in which a graph is embedded. On the other hand, the topological distance between sites $i$ and $j$, denoted by $d_{i j}$ hereafter, is the smallest number of links joining $i$ and $j$. In this paper we only use the topological properties and distances of the Bethe lattice. Note that the Bethe lattice can be embedded in a hyperbolic (Lobachevsky) space with metric properties different from Euclidian space. ${ }^{43}$

We now consider hopping Hamiltonians on an arbitrary, infinite graph; for simplicity we assume at most one link between two sites and no loops of length one. In terms of the quantum-mechanical single-particle operator $|i\rangle\langle j|$, which removes a particle from site $j$ and recreates it at site $i$, the general tight-binding hopping Hamiltonian can be written as

$$
H=\sum_{i, j \in V} t_{i j}|i\rangle\langle j|=\sum_{d \geqslant 0} t_{d} H_{d}
$$

where

$$
H_{d}=\sum_{\substack{i, j \in V \\ d_{i j}=d}}|i\rangle\langle j|
$$

describes hopping between sites $i$ and $j$ separated by topological distance $d$, i.e., $t_{i j}=t_{d_{i j}}$. By definition all nonzero matrix elements of $H_{1}$ are equal to 1 , i.e.,

$$
\left(H_{1}\right)_{i j}=\left\langle i\left|H_{1}\right| j\right\rangle= \begin{cases}1 & \text { if } d_{i j}=1, \\ 0 & \text { otherwise, }\end{cases}
$$

and $H_{0}=\sum_{i}|i\rangle\langle i|=1$ is the identity.

The topological properties of a graph are completely described by the nearest-neighbor hopping Hamiltonian $H_{1}$. This concept is used in graph theory where $H_{1}$ is called $a d$ - 
jacency matrix ${ }^{44}$ Furthermore, a simple interpretation can be given to the matrix elements of higher powers of the hopping Hamiltonian $H_{1}$, i.e., $\left(H_{1}^{n}\right)_{i j}$ is the number of paths connecting sites $i$ and $j$ in $n$ NN steps. ${ }^{44}$ Explicitly, one has

$$
\left(H_{1}^{n}\right)_{i j}=\sum_{k_{1}, \ldots, k_{n-1} \in V}\left(H_{1}\right)_{i k_{1}}\left(H_{1}\right)_{k_{1} k_{2}} \cdots\left(H_{1}\right)_{k_{n-1} j},
$$

where a term in the sum on the right-hand side is equal to unity if the string of indices $\left(i k_{1} k_{2} \cdots k_{n-1} j\right)$ represents a path joining $i$ and $j$, and is zero otherwise. Hence, $\left(H_{1}^{n}\right)_{i j}$ is equal to the number of paths connecting $i$ and $j$ with $n$ NN steps.

On the Bethe lattice the quantity $\left(H_{1}^{n}\right)_{i j}$ turns out to be a function only of $n$ and of the topological distance $d_{i j}$, while the specific positions of $i$ and $j$ are unimportant. Graphs with this property are called distance regular in graph theory. ${ }^{45}$ The triangular Husimi cactus, which is a set of triangles connected by vertices (see the Appendix), is also distance regular, and hence this property does not depend on the absence of loops. However, the situation is different for periodic (Bravais) lattices. For example, given a site $i$ on the twodimensional square lattice, there are two different positions for a next-nearest-neighbor site $\left(d_{i j}=2\right)$, one along an axis, the other on the diagonal of a plaquette. In the former case there is only one path of length two between $i$ and $j$, whereas in the latter case there are two paths. Thus for crystal lattices not only topological distances but also specific positions play a role in determining $H_{1}^{n}$.

For distance-regular graphs $H_{d}$ can be written in terms of powers of $H_{1} \cdot{ }^{45}$ We show this by first proving the inverse relation, i.e.,

$$
\left(H_{1}\right)^{n}=\sum_{d=0}^{n} a_{n}^{(d)} H_{d}
$$

where $a_{n}^{(d)}$ is the number of paths with $n$ NN steps between sites separated by a topological distance $d$. This relation can be easily verified by calculating the corresponding matrix elements

$$
\left(H_{1}^{n}\right)_{i j}=\sum_{d=0}^{n} a_{n}^{(d)}\left(H_{d}\right)_{i j}
$$

Using $\left(H_{d}\right)_{i j}=\delta_{d, d_{i j}}$ one finds $\left(H_{1}^{n}\right)_{i j}=a_{n}^{\left(d_{i j}\right)}$, which indeed agrees with Eq. (3), hence proving (4). Since $a_{n}^{(n)} \neq 0$ for all $n$, and $a_{n}^{(d)}=0$ if $d>n$, this (triangular) system of equations can always be inverted, yielding

$$
H_{d}=\sum_{n=0}^{d} A_{d}^{(n)}\left(H_{1}\right)^{n}
$$

Therefore for distance-regular graphs the hopping Hamiltonians $H_{d}$ are given by polynomials in $H_{1}$ of order $d$. For example, for a Bethe lattice with coordination number $Z$ the first few equations (4) read

$$
\begin{gathered}
\left(H_{1}\right)^{2}=Z 1+H_{2}, \\
\left(H_{1}\right)^{3}=(2 Z-1) H_{1}+H_{3},
\end{gathered}
$$

$$
\left(H_{1}\right)^{4}=Z(2 Z-1) 1+(3 Z-2) H_{2}+H_{4} .
$$

By contrast, such relations do not exist for all path lengths $n$ and topological distances $d$ on periodic lattices, although low-order relations may be found for certain graphs. For example, for the honeycomb lattice, which is similar to a Bethe lattice with $Z=3$ when taking at most two NN steps, $H_{1}^{2}$ can be related to $H_{1}$ and $H_{2}$ as in Eq. (7a).

\section{OPERATOR IDENTITIES FOR BETHE LATTICES}

We now determine the coefficients in Eqs. (4) and (6) for the Bethe lattice. These equations can be summarized as an operator identity involving the hopping Hamiltonians $H_{d}$. As an application the Green function for NN hopping is obtained.

\section{A. Recursive relations and generating function}

We consider a Bethe lattice with coordination number $Z \geqslant 2$, i.e., branching ratio $K=Z-1 \geqslant 1$. In this case the coefficients $a_{n}^{(d)}$ in (4) are obtained from a simple recursion, starting from $a_{0}^{(0)}=a_{1}^{(1)}=1$. Namely, each path of length $n \geqslant 1$ joining a site $i$ to a different site $j$ (with $d=d_{i j} \geqslant 1$ ) is composed of a path that joins $i$ to some nearest neighbor of $j$ within $n-1$ steps and one final step to $j$. Since for the last step there is only one possibility, $a_{n}^{(d)}$ is given by the number of possible paths with $(n-1)$ steps between $i$ and the nearest neighbors of $j$. Of the $Z$ nearest-neighbor sites of $j, Z-1$ are separated from $i$ by a distance $d+1$ and one by distance $d-1$. Together with a similar argument for $d=0$ we thus have the recurrence relations

$$
\begin{gathered}
a_{n}^{(d)}=K a_{n-1}^{(d+1)}+a_{n-1}^{(d-1)}, \quad d \geqslant 1, \\
a_{n}^{(0)}=Z a_{n-1}^{(1)}, \\
a_{n}^{(n)}=1,
\end{gathered}
$$

where the last equation is due to the treelike structure of the Bethe lattice. Since on the Bethe lattice it is impossible to return to the same site within an odd number of steps, we note that $a_{n}^{(d)}$ vanishes if $n+d$ is odd.

These recursion relations for the Bethe lattice can be solved in closed form. One first considers the generating functions $F_{d}(u)=\sum_{n=0}^{\infty} a_{n}^{(d)} u^{n}$, which appear when summing over Eq. (4), i.e., $\left[1-u H_{1}\right]^{-1}=\sum_{d=0}^{\infty} H_{d} F_{d}(u)$. They obey the recursion relations $F_{d}(u)=K u F_{d+1}(u)+u F_{d-1}(u)$ for $d \geqslant 1$ and $F_{0}(u)=1+u Z F_{1}(u)$. These equations may be solved by the ansatz $^{46} F_{d}(u)=f(u)[u g(u)]^{d}$, which yields $g(u)$ $=2 /\left(1+\sqrt{1-4 K u^{2}}\right)$ and $f(u)=\left[1-Z u^{2} g(u)\right]^{-1}$, where the sign in front of the square root has been chosen so that $F_{d}(u)$ $=u^{d}[1+O(u)]$ is satisfied. By setting $x=u g(u)$ and rearranging the terms we finally obtain the remarkable operator identity 


$$
\frac{1-x^{2}}{1-x H_{1}+K x^{2}}=\sum_{d=0}^{\infty} H_{d} x^{d} .
$$

From this formal power series the coefficients in Eqs. (4) and (6) can be extracted in closed form. After some algebra we find that the nonzero coefficient $a_{n}^{(d)}$ and $A_{n}^{(d)}$ are given by

$$
\begin{aligned}
& a_{n}^{(n-2 s)}=\sum_{r=0}^{s}\left[\left(\begin{array}{l}
n \\
r
\end{array}\right)-\left(\begin{array}{c}
n \\
r-1
\end{array}\right)\right] K^{r}, \\
& A_{n}^{(n-2 s)}=(-1)^{s} \sum_{r=0}^{1}\left(\begin{array}{c}
n-s-r \\
s-r
\end{array}\right) K^{s-r},
\end{aligned}
$$

for $0 \leqslant 2 s \leqslant n$. By induction with respect to $n$ it is also straightforward to verify that Eq. (10) is the solution of the recursion relations (8).

Note that in the limit of infinite connectivity, $K \rightarrow \infty$ (or $Z \rightarrow \infty)$, one must scale ${ }^{24} H_{d}$ by $Z_{d}^{-1 / 2}$, where $Z_{d}$ is the number of sites that a given site is connected to by $H_{d}$. To leading order one has $Z_{d} \sim Z^{d} \sim K^{d}$; thus we introduce scaled operators, $\widetilde{H}_{d}:=H_{d} / K^{d / 2}$. By computing coefficients we then obtain from Eq. (9) the relation

$$
\begin{gathered}
\tilde{H}_{d}=U_{d}\left(\tilde{H}_{1} / 2\right)-\frac{1-\delta_{d 0}-\delta_{d 1}}{K} U_{d-2}\left(\tilde{H}_{1} / 2\right), \\
U_{n}\left(\tilde{H}_{1} / 2\right)=\sum_{s=0}^{\lfloor n / 2\rfloor} \frac{\tilde{H}_{n-2 s}}{K^{s}},
\end{gathered}
$$

which are valid for any $K \geqslant 1$ and reduce to $\tilde{H}_{d}=U_{d}\left(\tilde{H}_{1} / 2\right)$ in the limit $K \rightarrow \infty$. Here $U_{n}(x)$ are the Chebyshev polynomials of the second kind, ${ }^{47}$ with generating function $\left(1-2 x t+t^{2}\right)^{-1}=\sum_{n=0}^{\infty} U_{n}(x) t^{n}$. It seems that the operator identities (9) and (12) might also be useful in other contexts involving the Bethe lattice. Note that these relations remain valid for the one-dimensional chain $(K=1)$. For the triangular Husimi cactus a relation similar to (9) is derived in the Appendix.

\section{B. Green function}

As a first application of the operator identity (9) we present a shortcut to compute the Green function for the NN hopping Hamiltonian $t_{1} H_{1}$ on the Bethe lattice,

$$
G_{i j}(z)=\left\langle i\left|\left(z-t_{1} H_{1}\right)^{-1}\right| j\right\rangle,
$$

with $\operatorname{Im} z \neq 0$. This will fit into Eq. (9) if we set $z=t_{1}\left(1+K x^{2}\right) / x$, i.e.,

$$
x=\frac{2 t_{1}}{z+\sqrt{z^{2}-4 K t_{1}^{2}}},
$$

with $\operatorname{sgn}\left(\operatorname{Im} \sqrt{z^{2}-4 K t_{1}^{2}}\right)=\operatorname{sgn}(\operatorname{Im} z)$. Since for the Bethe lattice there is only one non-self-intersecting path connecting sites $i$ and $j$ we have $\left\langle i\left|H_{d}\right| j\right\rangle=\delta_{d, d_{i j}}$, whence

$$
\begin{aligned}
G_{i j}(z) & =\left\langle i\left|\left(\frac{1}{t_{1}} \frac{x}{1-x^{2}} \sum_{d=0}^{\infty} H_{d} x^{d}\right)\right| j\right\rangle=\frac{1}{t_{1}} \frac{x^{d_{i j}+1}}{1-x^{2}} \\
& =\frac{2 K}{(K-1) z+Z \sqrt{z^{2}-4 K t_{1}^{2}}}\left(\frac{2 t_{1}}{z+\sqrt{z^{2}-4 K t_{1}^{2}}}\right)^{d_{i j}}
\end{aligned}
$$

in agreement with Refs. 21 and 22. This yields the wellknown expression for the DOS of $\widetilde{H}_{1}$,

$$
\rho_{1}(\lambda)=-\frac{1}{\pi} \operatorname{Im} G_{i i}(\lambda+i 0)=\frac{1}{2 \pi} \frac{\sqrt{4-\lambda^{2}}}{p-\lambda^{2} / Z},
$$

where we set $\left|t_{1}\right|=1 / \sqrt{K}$ and $p=Z / K=1+1 / K$. In the limit $Z \rightarrow \infty$ this leads to the familiar semielliptic density of states,

$$
\rho_{1}^{\infty}(\lambda):=\lim _{K \rightarrow \infty} \rho_{1}(\lambda)=\frac{\sqrt{4-\lambda^{2}}}{2 \pi},
$$

as mentioned in the introduction, with half-bandwidth $D=2$. For brevity, here and below we use the convention that any square root that appears in a DOS yields zero if its argument is negative.

\section{DENSITY OF STATES FOR ARBITRARY-RANGE HOPPING}

The DOS encodes information about hopping parameters and underlying lattice structure. For general tight-binding Hamiltonians on the Bethe lattice we now derive an expression for the DOS by using the operator identities of the preceding section. In particular for $t_{1}-t_{2}$ hopping we explicitly evaluate the DOS and the local Green function. The case of exponentially decreasing hopping is also discussed. Furthermore we show how to construct a tight-binding Hamiltonian from a given DOS.

\section{A. Dispersion relations for tight-binding Hamiltonians}

Since the hopping Hamiltonian $H_{d}$ on the Bethe lattice is a polynomial in $\widetilde{H}_{1}$ [see Eq. (12a)], its eigenfunctions are the same as those of $\tilde{H}_{1}$, and its eigenvalues can be expressed as a function of the eigenvalues of $\widetilde{H}_{1}$. Explicitly, for a general tight-binding Hamiltonian of the form (1), i.e.,

$$
H=\sum_{d=0}^{\infty} t_{d} H_{d}=\sum_{d=0}^{\infty} t_{d}^{*} \tilde{H}_{d}
$$

we find from Eq. (12a) that $H=\mathcal{F}\left(\tilde{H}_{1}\right)$ with

$$
\mathcal{F}(x)=\sum_{d=0}^{\infty}\left(t_{d}^{*}-\frac{t_{d+2}^{*}}{K}\right) U_{d}(x / 2),
$$

where we used again the scaling $t_{d}=t_{d}^{*} / K^{d / 2}$ and $\widetilde{H}_{d}=H_{d} / K^{d / 2}$ to facilitate the discussion of the limit $Z \rightarrow \infty$. The eigenvalues $\epsilon$ of $H$ are thus related to the eigenvalues $\lambda$ of $\widetilde{H}_{1}$ by the "dispersion relation" 


$$
\epsilon(\lambda)=\mathcal{F}(\lambda),
$$

which provides a surprising analogy to tight-binding dispersions for crystal lattices with translational symmetries, with $\lambda$ playing the role of crystal momentum. As a consequence of Eq. (20) the DOS for $H$ can be obtained by a simple change of variables from that of $\tilde{H}_{1}$ [see Eq. (16)],

$$
\begin{aligned}
\rho(\epsilon) & =\int_{-2}^{2} \rho_{1}(\lambda) \delta[\epsilon-\epsilon(\lambda)] d \lambda \\
& =\sum_{i}\left|\lambda_{i}^{\prime}(\epsilon)\right| \rho_{1}\left[\lambda_{i}(\epsilon)\right],
\end{aligned}
$$

where the sum runs over all solutions $\lambda_{i}(\epsilon)$ of $\epsilon=\epsilon(\lambda)$. Explicit eigenfunctions $|\theta\rangle$ and eigenvalues $\lambda(\theta)$ of $\widetilde{H}_{1}$ were obtained by Mahan in Ref. 23.

We see that using Eqs. (18)-(22) the DOS can be easily obtained for any tight-binding Hamiltonian. At most a simple numerical inversion of the polynomial $\epsilon(\lambda)$ needs to be performed. Below we discuss the case of $t_{1}-t_{2}$ hopping as well as an exemplary case of long-range hopping, for which this inversion can be performed analytically.

\section{B. $t_{1}-t_{2}$ hopping}

The DOS for the Hamiltonian with nearest-neighbor $\left(t_{1}\right)$ and next-nearest-neighbor $\left(t_{2}\right)$ hopping can be found from

$$
H_{t_{1}, t_{2}^{*}}^{*}:=t_{1} H_{1}+t_{2} H_{2}=t_{1}^{*} \tilde{H}_{1}+t_{2}^{*} \tilde{H}_{2}=t_{2}^{*} \tilde{H}_{1}^{2}+t_{1}^{*} \widetilde{H}_{1}-p t_{2}^{*} \rrbracket,
$$

where again $p=Z / K$, and $t_{1}=t_{1}^{*} / \sqrt{K}$ and $t_{2}=t_{2}^{*} / K$ were scaled appropriately, such that the limit $Z \rightarrow \infty$ can be taken. The dispersion relation and the roots are given by

$$
\begin{gathered}
\epsilon(\lambda)=t_{2}^{*} \lambda^{2}+t_{1}^{*} \lambda-t_{2}^{*} p, \\
\lambda_{1,2}(\epsilon)=\frac{-t_{1}^{*} \pm \sqrt{t_{1}^{* 2}+4 t_{2}^{*}\left(p t_{2}^{*}+\epsilon\right)}}{2 t_{2}^{*}} .
\end{gathered}
$$

This yields the DOS

$$
\begin{gathered}
\rho_{t_{1}^{*}, t_{2}^{*}}(\epsilon)=\frac{1}{2 \pi} \frac{\Theta\left[t_{1}^{* 2}+4 t_{2}^{*}\left(p t_{2}^{*}+\epsilon\right)\right]}{\sqrt{t_{1}^{* 2}+4 t_{2}^{*}\left(p t_{2}^{*}+\epsilon\right)}} \sum_{i=1}^{2} \frac{\sqrt{4-\lambda_{i}(\epsilon)^{2}}}{p-\lambda_{i}(\epsilon)^{2} / Z}, \\
=\rho_{-t_{1}^{*}, t_{2}^{*}}(\epsilon)=\rho_{t_{1}^{*},-t_{2}^{*}}(-\epsilon),
\end{gathered}
$$

where $\Theta(x)$ denotes the step function. In the limit $Z \rightarrow \infty$ this simplifies to

$$
\begin{gathered}
\rho_{t_{1}^{*}, t_{2}^{*}}^{\infty}(\epsilon)=\frac{\Theta\left[t_{1}^{* 2}+4 t_{2}^{*}\left(t_{2}^{*}+\epsilon\right)\right]}{\sqrt{t_{1}^{* 2}+4 t_{2}^{*}\left(t_{2}^{*}+\epsilon\right)}} \sum_{i=1}^{2} \frac{\sqrt{4-\lambda_{i}^{\infty}(\epsilon)^{2}}}{2 \pi}, \\
\lambda_{1,2}^{\infty}(\epsilon)=\frac{-t_{1}^{*} \pm \sqrt{t_{1}^{* 2}+4 t_{2}^{*}\left(t_{2}^{*}+\epsilon\right)}}{2 t_{2}^{*}} .
\end{gathered}
$$

Note that for $t_{2}^{*} \neq 0$ the DOS is asymmetric, ${ }^{35}$ in contrast to

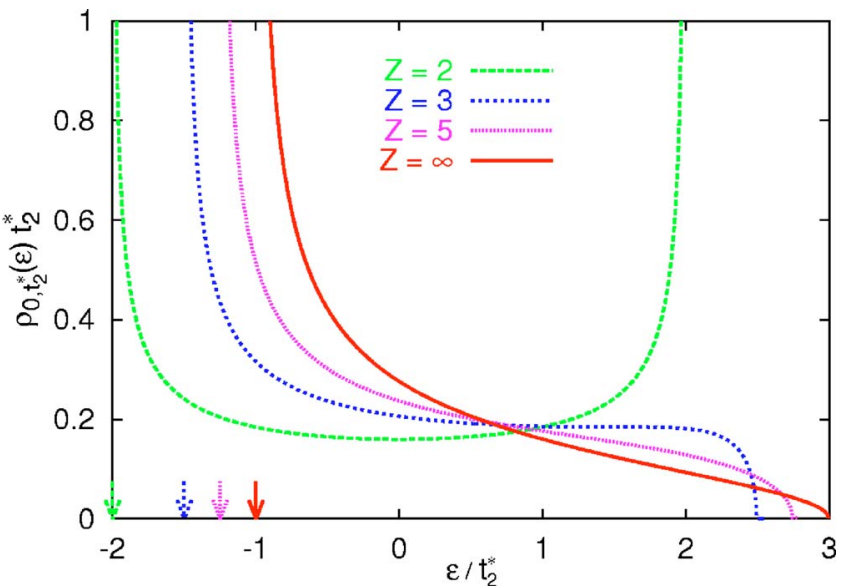

FIG. 2. (Color online) Density of states (30) for pure NNN hopping $t_{2}=t_{2}^{*} /(Z-1) \neq 0$ on the Bethe lattice, for several numbers of nearest neighbors $Z ; t_{2}^{*}=1$ sets the energy scale. Divergences are marked on the horizontal axis.

the case of random $t_{1}-t_{2}$ hopping on the Bethe lattice. ${ }^{29,36}$

We now discuss some limiting cases of Eqs. (26) and (28). For $t_{2}^{*} \rightarrow 0$ (pure NN hopping) we recover Eq. (16) since then one of the roots $\lambda_{i}$ reduces to $\epsilon / t_{1}^{*}$ while the other diverges. On the other hand, for $t_{1}^{*} \rightarrow 0$ the DOS for pure NNN hopping (with $\left|t_{2}^{*}\right|=1$ ) is obtained,

$$
\rho_{0,1}(\epsilon)=\frac{1}{2 \pi} \frac{\sqrt{4-p-\epsilon}}{(1-\epsilon / Z) \sqrt{p+\epsilon}}=\rho_{0,-1}(-\epsilon) .
$$

In the limit $Z \rightarrow \infty$ this reduces to $^{35}$

$$
\rho_{0,1}^{\infty}(\epsilon)=\frac{1}{2 \pi} \frac{\sqrt{3-\epsilon}}{\sqrt{1+\epsilon}}=\rho_{0,-1}^{\infty}(-\epsilon) .
$$

In Fig. $2 \rho_{0, t_{2}^{*}}(\epsilon)$ (pure NNN hopping) is plotted for several values of $Z$. This function has a square-root singularity on the left-hand side of the band. In the case $Z=2$ the DOS has the same form as for a chain with NN hopping, because then the Bethe lattice with NNN hopping reduces to two disconnected infinite chains. The DOS for $t_{1}-t_{2}$ hopping on the triangular Husimi cactus is obtained in the Appendix.

In general, the shape of $\rho_{t_{1}^{*}, t_{2}^{*}}^{*}(\epsilon)$ is determined by two dimensionless parameters, the coordination number $Z$ and the ratio $t_{2}^{*} / t_{1}^{*}$. In view of Eq. (27) it is sufficient to consider $t_{1}^{*}, t_{2}^{*} \geqslant 0$. In the following we use the parameter $x=t_{2}^{*} /\left(t_{1}^{*}+t_{2}^{*}\right) \in[0,1]$, i.e., $t_{2}^{*}=x\left(t_{1}^{*}+t_{2}^{*}\right), t_{1}^{*}=(1-x)\left(t_{1}^{*}+t_{2}^{*}\right)$. In Figs. 3 and 4 we present plots of $\rho_{t_{1}^{*}, t_{2}^{*}}(\epsilon)$ for several values of the parameter $x$ in the case $Z=4$ and in the limiting case $Z \rightarrow \infty$. At small $x\left(t_{1}^{*} \gg t_{2}^{*}\right)$ the asymmetry of the DOS develops gradually. At some critical value $x_{*}=1 / 5$ a square-root singularity appears at the left-hand side of the band. For $x<x_{*}$ the DOS is a smooth function vanishing at both band edges, whereas for $x>x_{*}$ there is a singularity at the lower band edge as well as a cusp within the band with diverging first derivative $d \rho_{t_{1}}^{*}, t_{2}^{*}(\epsilon) / d \epsilon$. With increasing $x$ the cusp moves continuously to the upper band edge where it disappears for $x=1$. These features can be understood with the 

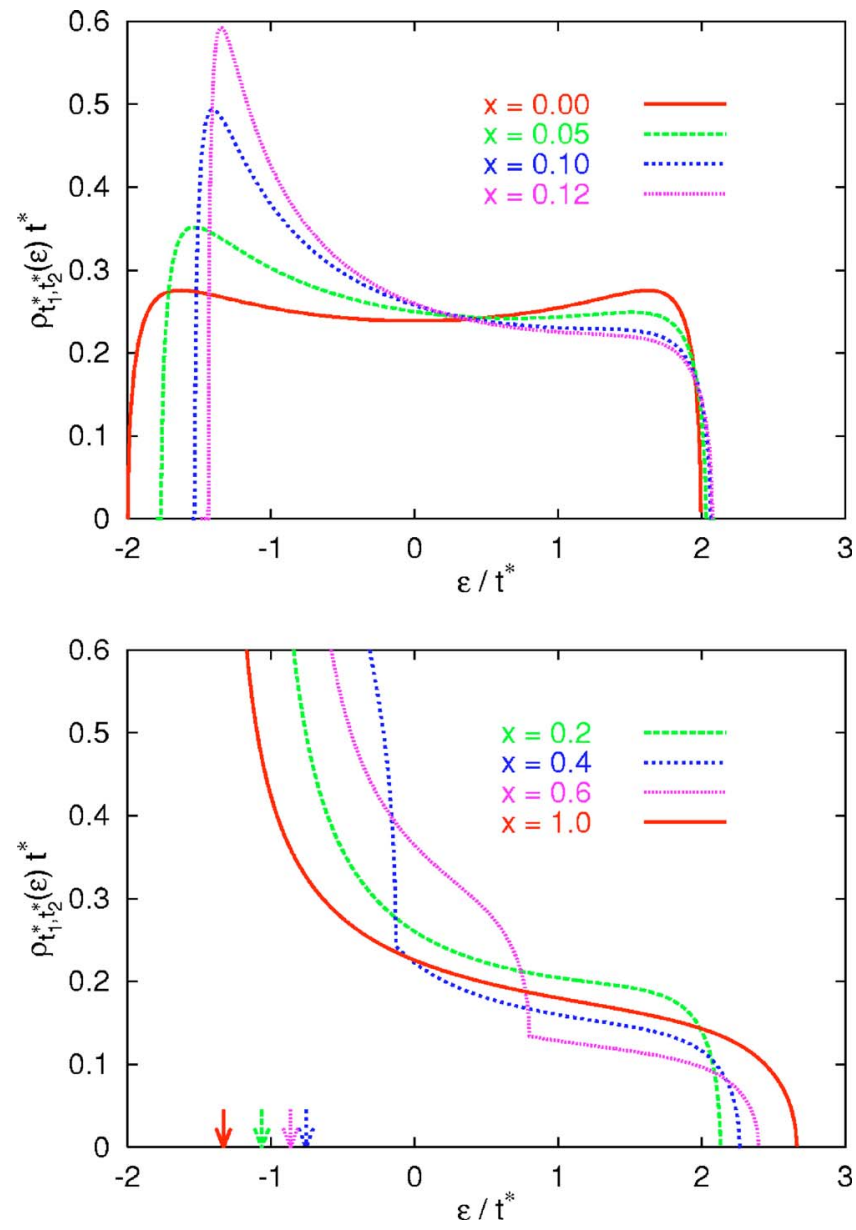

FIG. 3. (Color online) Density of states (26) for $t_{1}-t_{2}$ hopping on the Bethe lattice with $Z=4$ for selected values of $x=t_{2}^{*} / t^{*}$, where $t_{1}^{*}, t_{2}^{*} \geqslant 0, t^{*}=t_{1}^{*}+t_{2}^{*}$ sets the energy scale. Divergences are marked on the horizontal axis.

help of the dispersion relation Eq. (24). The solutions $\lambda_{1,2}$ [Eq. (25)] of Eq. (24) contribute only if they lie in the interval $[-2,2]$ of nonvanishing $\rho_{1}(\lambda)$. While for $x<x_{*}$ only $\lambda_{1}$ contributes, for $x>x *$ both solutions contribute if $\epsilon$ lies between the left band edge and the cusp, whereas only one solution contributes if $\epsilon$ lies above the cusp.

Finally we note that the local Green function can be obtained from the DOS by a Kramers-Kronig relation, i.e.,

$$
G_{t_{1}^{*}, t_{2}^{*}}^{\infty}(z)=\int_{-\infty}^{\infty} \frac{\rho_{t_{1}^{*}, t_{2}^{*}}^{*}(\epsilon)}{z-\epsilon} d \epsilon .
$$

For the case $Z \rightarrow \infty$ this leads to the result

$$
\begin{gathered}
G_{t_{1}^{*}, t_{2}^{*}}^{\infty}(z)=\left\langle i\left|\left(z-t_{1}^{*} \tilde{H}_{1}-t_{2}^{*} \tilde{H}_{2}\right)^{-1}\right| i\right\rangle \\
=t_{2}^{*} \frac{G_{t_{2}^{*}, 0}^{\infty}\left(z_{1}\right)-G_{t_{2}^{*}, 0}^{\infty}\left(z_{2}\right)}{z_{1}-z_{2}}
\end{gathered}
$$

where $\quad z_{1,2}=\left[-t_{1}^{*} \pm \sqrt{t_{1}^{* 2}+4 t_{2}^{*}\left(z+t_{2}^{*}\right)}\right] / 2$ and $G_{t^{*}, 0}^{\infty}(z)$ $=\left(z-\sqrt{z^{2}-4 t^{* 2}}\right) /\left(2 t^{* 2}\right)$ is the usual local Green function for
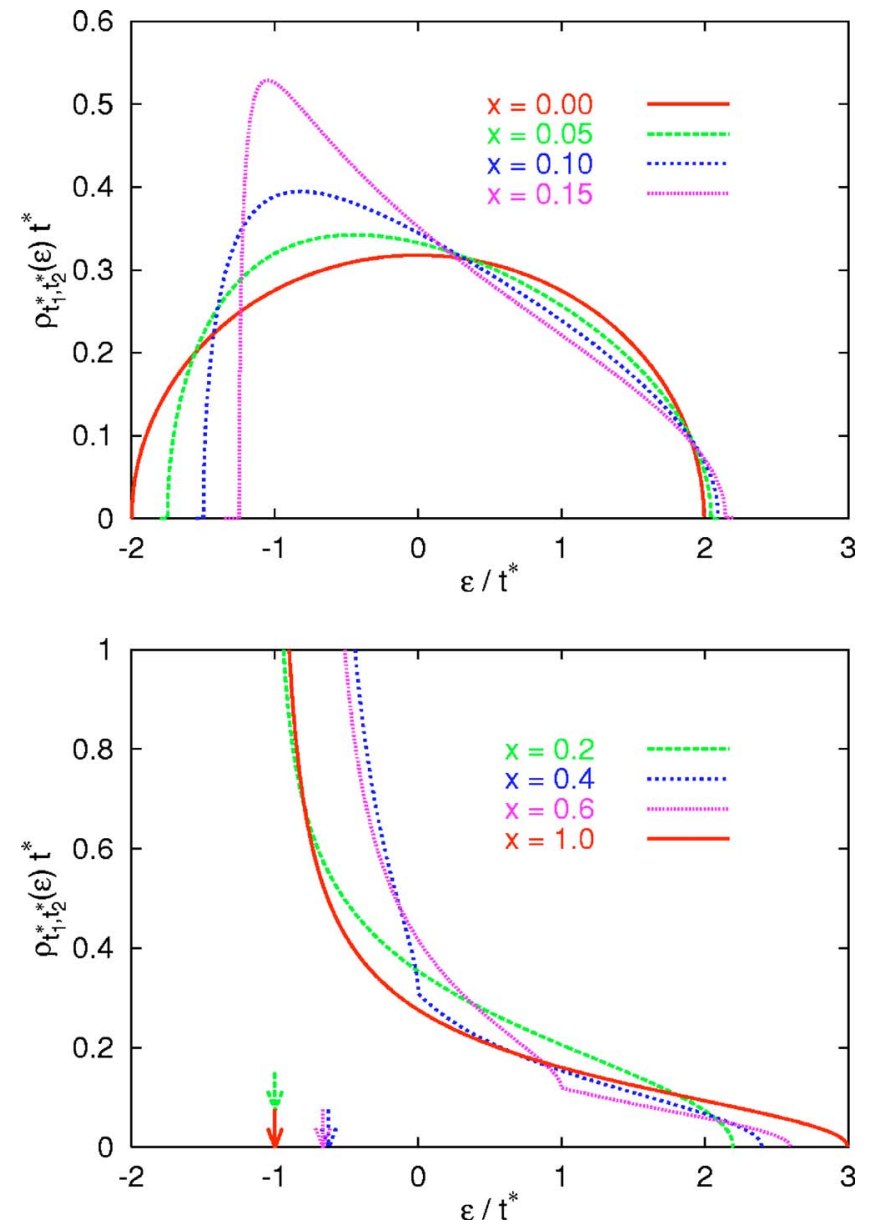

FIG. 4. (Color online) Same as Fig. 3, but for $Z \rightarrow \infty$ [Eq. (28)].

pure NN hopping as obtained from (15). For pure $t_{2}$ hopping (34) reduces to

$$
G_{0, t_{2}^{*}}^{\infty}(z)=\frac{1}{2 t_{2}^{*}}-\frac{1}{t_{2}^{*}} \sqrt{\frac{1}{4}-\frac{1}{1+z / t_{2}^{*}}},
$$

with the square root given by its principal branch. The density of states and Green function for pure NNN hopping [Eqs. (31) and (35)] were previously obtained in Ref. 35 using RPE.

\section{Long-range hopping}

For long-range hopping beyond NNN the expressions for $\lambda(\epsilon)$ and $\rho(\epsilon)$ become rather complicated. However, the special case of exponentially decreasing hopping amplitudes, $t_{d}=w^{d-1} t^{*}$, with Hamiltonian

$$
H_{w}=t^{*} \sum_{d=1}^{\infty} w^{d-1} \tilde{H}_{d},
$$

allows for an analytical solution (here we assume $|w|<1$ to ensure convergence). The familiar case of pure $t_{1}$ hopping corresponds to $w \rightarrow 0$. From Eq. (9) it is straightforward to obtain the corresponding dispersion relation as 


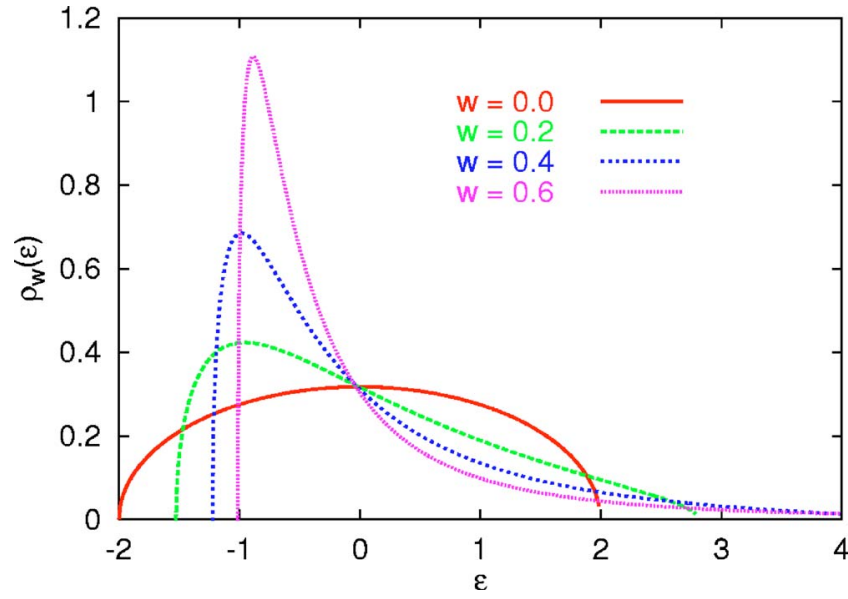

FIG. 5. (Color online) Density of states for exponentially decreasing long-range hopping on the Bethe lattice [Eq. (36)], for $t^{*}=1$ and $Z \rightarrow \infty$.

$$
\epsilon_{w}(\lambda)=\frac{t^{*}}{w}\left(\frac{1-w^{2} / K}{1-w \lambda+w^{2}}-1\right),
$$

i.e., $\epsilon_{w}(\lambda)=t^{*} \lambda+O(w)$. In the interval $-2 \leqslant \lambda \leqslant 2, \epsilon_{w}(\lambda)$ is a monotonous function with inverse function $\lambda_{w}(\epsilon)$. The DOS $\rho(\epsilon)$ is again calculated using Eq. (22),

$$
\rho_{w}(\epsilon)=\frac{t^{*}}{2 \pi} \frac{1-w^{2} / K}{\left(w \epsilon+t^{*}\right)^{2}} \frac{\sqrt{4-\lambda_{w}(\epsilon)^{2}}}{p-\lambda_{w}(\epsilon)^{2} / Z},
$$

where again $p=Z / K=1+1 / K$. In Fig. $5 \rho_{w}(\epsilon)$ is plotted for several values of $w$ in the limit $Z \rightarrow \infty$. For $|w|<1$ we find a smooth DOS with finite bandwidth, while for $w \rightarrow 1$ the upper band edge [where $\lambda_{w}(\epsilon)=2$ ] moves to infinity and the DOS decreases like $\epsilon^{-5 / 2}$ for large $\epsilon$.

It is also interesting to consider only "odd" hopping, i.e., between different $A$ and $B$ sublattices, or "even" hopping between the same sublattices, although there is no immediate physical motivation for this restriction. The Hamiltonians $H_{w}^{-}=t^{*} \Sigma_{d=1}^{\infty} w^{d-1} \hat{H}_{2 d-1}$ and $H_{w}^{+}=t^{*} \sum_{d=1}^{\infty} w^{d-1} \hat{H}_{2 d}$ describe odd and even hopping with exponentially decreasing amplitudes, respectively. They lead to the dispersion relations

$$
\begin{gathered}
\boldsymbol{\epsilon}_{w}^{-}(\lambda)=t^{*} \frac{(1-w / K) \lambda}{(1+w)^{2}-w \lambda^{2}}, \\
\epsilon_{w}^{+}(\lambda)=\frac{t^{*}}{w}\left(\frac{(1+w)(1-w / K)}{(1+w)^{2}-w \lambda^{2}}-1\right),
\end{gathered}
$$

which are antisymmetric and symmetric in $\lambda$, respectively, and yield finite bandwidths except for $w=1$. Thus for odd hopping the DOS $\rho_{w}^{-}(\epsilon)$ is symmetric as in Fig. 6, whereas for even hopping $\rho_{w}^{+}(\epsilon)$ is asymmetric (Fig. 7). We note that $\rho_{w}^{-}(\epsilon)$ is finite for $w>w_{*}:=3-2 \sqrt{2} \approx-0.172$, while squareroot singularities at the band edges occur for $w<w_{*}$. For the latter case another remarkable feature is that the DOS is nearly constant in the middle of the band, up to a cusp where it rises sharply (see Fig. 6). On the other hand, $\rho_{w}^{+}(\epsilon)$ always has a square-root singularity at one band edge

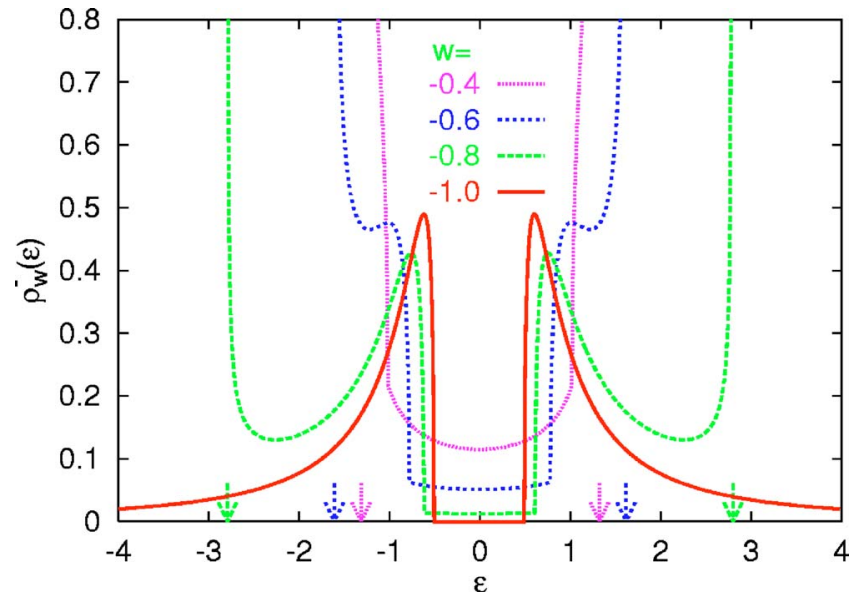

FIG. 6. (Color online) Density of states for exponentially decreasing long-range hopping between different sublattices ("odd hopping") for $t^{*}=1, Z \rightarrow \infty$, and $w<w_{*}$. Divergences are marked on the horizontal axis.

$\left[\epsilon=-p t^{*} /(1+w)\right]$, similar to the case of pure NNN hopping. Note that for $w \rightarrow-1$ this singularity moves to infinity albeit with vanishing weight; most of the weight is near the other band edge in this limit.

We note that by naively putting $w=\sqrt{K}$ (without addressing questions of convergence) in the above results one recovers the case of infinite-range hopping, where each site is connected to every other site ("complete graph") and the underlying lattice structure becomes irrelevant. The energy $\epsilon(\lambda)$ becomes constant in this case and we find $\rho_{w}(\epsilon) \rightarrow \delta\left(\epsilon+t^{*} / \sqrt{K}\right)$, in agreement with the result for infinite-range hopping on the hypercubic lattice, for which the corresponding Hubbard model is solvable. ${ }^{48}$

\section{Tight-binding Hamiltonian for arbitrary density of states}

In mean-field theories the dependence on the lattice typically enters only through the DOS for free particles. For

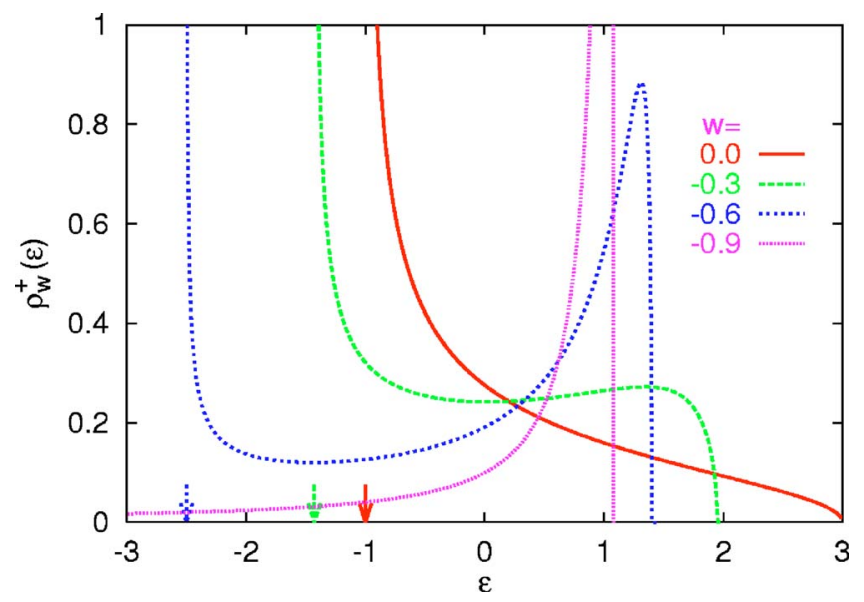

FIG. 7. (Color online) Density of states for exponentially decreasing long-range hopping between same sublattices ("even hopping") for $t^{*}=1, Z \rightarrow \infty$, and $w \leqslant 0$. Divergences are marked on the horizontal axis. For $w=-0.9$ the finite peak at the upper band edge, as well as the singularity at the lower band edge, are outside the plotting range. 


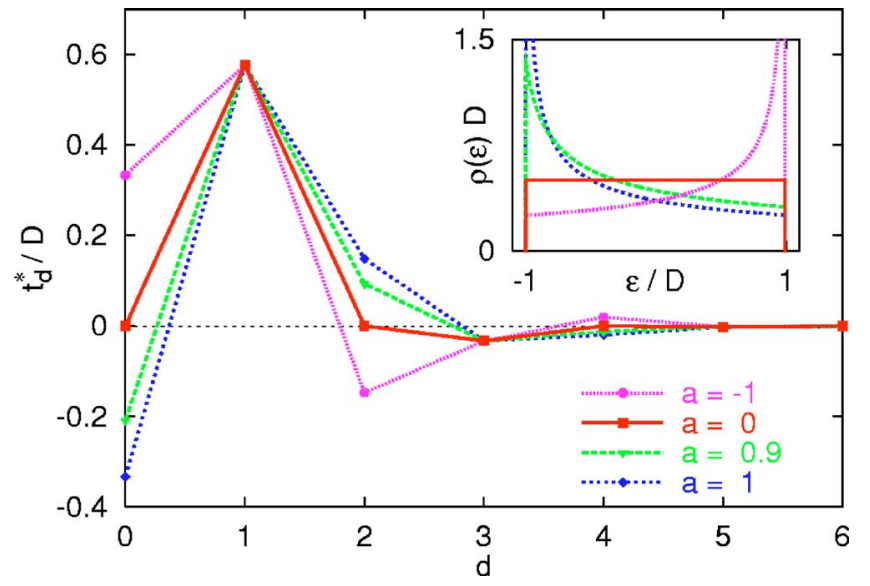

FIG. 8. (Color online) Hopping amplitudes $t_{d}^{*}$ on the Bethe lattice (with $Z \rightarrow \infty$ ) corresponding to the DOS (44) (see inset) for several values of the asymmetry parameter $a$. The odd hopping amplitudes $t_{1}^{*}, t_{3}^{*}$, etc., are independent of $a ; t_{0}^{*}$ gives the center of the mass of the band.

simplicity often DOSs with a particularly simple form are employed, but when calculating two-particle quantities or going beyond mean-field theory it is necessary to know the corresponding hopping amplitudes. They are also useful for the realistic modelling of strongly correlated materials where the DOS is obtained from $a b$ initio calculations. ${ }^{30}$ In this section we show how to determine a tight-binding Hamiltonian $H$ that corresponds to a given (one-band) DOS $\rho(\epsilon)$. For hypercubic lattices this task has already been addressed in Refs. 35 and 49-51. Here we perform such a construction for the Bethe lattice, based on the relation (9).

We start again from the general hopping Hamiltonian (18). As noted above, the eigenvalues of $H$ are given by the dispersion relation $\epsilon(\lambda)=\mathcal{F}(\lambda)$ [see Eq. (19)]. We can thus use the methods of Blümer ${ }^{35,49}$ who found a similar map between the spectra of $H$ and $\widetilde{H}_{1}$ for the hypercubic lattice in the limit of high dimensions. In the present case we choose

$$
\mathcal{F}(\lambda)=\mu\left[n_{1}(\lambda)\right]
$$

where $n_{1}(\lambda)=\int_{-2}^{\lambda} \rho_{1}\left(\lambda^{\prime}\right) d \lambda^{\prime}$ and $n(\mu)=\int_{\epsilon_{\min }}^{\mu} \rho(\epsilon) d \epsilon$. Here $\epsilon_{\min }$ is the lower band edge of $\rho(\epsilon)$ and $\mu(n)$ denotes the inverse function of $n(\mu)$. Then $\mathcal{F}^{\prime}(\lambda)>0$ for $-2<\lambda<2$, and the DOSs are related by

$$
\rho_{1}(\lambda)=\mathcal{F}^{\prime}(\lambda) \rho[\mathcal{F}(\lambda)],
$$

which indeed implies Eq. (21). It remains to determine the hopping parameters in Eq. (18) for this choice of $\mathcal{F}(\lambda)$. Using the orthogonality of the Chebyshev polynomials ${ }^{47}$ we find that $t_{d+2}^{*} / K=t_{d}^{*}-u_{d}$, where

$$
u_{d}=\int_{-2}^{2} \rho_{1}^{\infty}(\lambda) \mathcal{F}(\lambda) U_{d}(\lambda / 2) d \lambda .
$$

For the remainder of this section we will consider only the limit $Z \rightarrow \infty$, for which we have $t_{d}^{*}=u_{d}$ and $n_{1}(\lambda)=[1$ $\left.+\lambda \rho_{1}^{\infty}(\lambda)\right] / 2+\arcsin (\lambda / 2) / \pi$, and $u_{0}=\int \epsilon \rho(\epsilon) d \epsilon=: M_{1}$ yields the first moment of the target DOS. Even then the calculation of the hopping amplitudes will typically involve numerical integrations. As an analytically tractable example we consider the model DOS,

$$
\rho(\epsilon)=c \frac{\Theta(D-|\epsilon|)}{\sqrt{1+a \epsilon / D}}, \quad c=\frac{\sqrt{1+a}+\sqrt{1-a}}{4 D},
$$

where $D$ is the half-bandwidth and the parameter $-1 \leqslant a$ $\leqslant 1$ determines the asymmetry, e.g., the first moment is given by $M_{1}=-a /\left(24 D c^{2}\right)$. A constant (rectangular) DOS is recovered for $a=0$, and a square-root singularity at one band edge is present for $|a|=1$. Note that this model DOS does not vanish at the band edges, in contrast to the DOS proposed in Ref. 51. For the latter, however, an analytical calculation of the corresponding hopping amplitudes appears infeasible.

For the DOS (44) we have $\mu(n)=-D+n / \rho(-D)-6 M_{1} n^{2}$. In general, upon expanding $\mu(n)$ as a power series in $n$, the calculation of $t_{d}^{*}$ involves the coefficients

$$
\begin{gathered}
r_{d, s}=\int_{-2}^{2} \rho_{1}^{\infty}(\lambda) U_{d}(\lambda / 2)\left[n_{1}(\lambda)\right]^{s} d \lambda \\
=\frac{2}{\pi} \int_{0}^{\pi} \sin x \sin (d+1) x\left(\frac{2 x-\sin 2 x}{2 \pi}\right)^{s} d x
\end{gathered}
$$

where we have used the representation ${ }^{47} U_{n}(x)$ $=\sin [(n+1) \arccos x] / \sin (\arccos x)$ for the Chebyshev polynomials. Evaluating Eq. (46) for $s=0,1,2$ we obtain for the DOS (44),

$$
\begin{gathered}
t_{d}^{*}= \begin{cases}D R(d) & \text { for odd } d, \\
-3 M_{1} R(d) & \text { for even } d,\end{cases} \\
R(d)= \begin{cases}\frac{-1}{3} & \text { for } d=0, \\
\frac{35}{24 \pi^{2}} & \text { for } d=2, \\
\frac{-7}{36 \pi^{2}} & \text { for } d=4, \\
\frac{\pi^{2}(d-2) d^{2}(d+2)^{2}(d+4)}{28(d+1)} & \text { otherwise. }\end{cases}
\end{gathered}
$$

In Fig. 8 we plot the hopping amplitudes $t_{d}^{*}$ vs $d$, which decay slower for large distances than in Eq. (36), due to the algebraic behavior of $R(d) \sim d^{-5}$. Note that the amplitudes for hopping between the same sublattices (even $d$ ) are proportional to $M_{1}=O(a)$ and thus vanish for the special case $a=0$ (constant DOS). On the other hand, for different sublattices (odd $d$ ) the hopping amplitudes are independent of the symmetry parameter $a$; except for $t_{1}^{*} \approx 0.58 D$ they are all negative and drop off quickly (e.g., $\left.t_{3}^{*}=14 t_{5}^{*} \approx-0.033 D\right)$. Regarding $t_{d}^{*} / t_{1}^{*}$ the quantitative difference between hopping for constant and semielliptic DOS (pure NN hopping) is thus surprisingly small. 


\section{DYNAMICAL MEAN-FIELD THEORY}

We now turn to dynamical mean-field theory (DMFT) ${ }^{25-30}$ for the Hubbard model, for which the Hamiltonian reads

$$
\begin{gathered}
H_{H}=H_{\mathrm{hop}}+H_{\mathrm{int}}+H_{\mathrm{ext}}, \\
H_{\mathrm{hop}}=\sum_{i j \sigma} t_{i j} c_{i \sigma}^{\dagger} c_{j \sigma}, \\
H_{\mathrm{int}}=U \sum_{i} n_{i \uparrow} n_{i \downarrow},
\end{gathered}
$$

where $c_{i \sigma}$ and $c_{i \sigma}^{\dagger}$ are the usual fermionic annihilation and creation operators for site $i$ and $\operatorname{spin} \sigma, n_{i \sigma}=c_{i \sigma}^{\dagger} c_{i \sigma}$ is number operator, and $H_{\text {ext }}$ involves external fields and is given below [Eq. (57)]. DMFT becomes exact for this model in the limit of infinite coordination number, $Z \rightarrow \infty .^{24}$ Below we will evaluate the DMFT equations for the Bethe lattice with arbitrary (in particular $t_{1}-t_{2}$ ) hopping.

\section{A. DMFT for the Hubbard model}

We begin with a very brief summary of DMFT for the Hubbard model (see Ref. 29 for a review). Let $\boldsymbol{G}$ and $\mathbf{\Sigma}$ denote the imaginary-time-ordered Green function and selfenergy, which are matrices in site labels $i$, spin indices $\sigma$, and imaginary-time slices $\tau$ (or Matsubara frequencies $i \omega_{n}$ ). They satisfy the Dyson equation

$$
\boldsymbol{G}=\left[\left(\boldsymbol{G}^{(0)}\right)^{-1}-\boldsymbol{\Sigma}\right]^{-1},
$$

where $\boldsymbol{G}^{(0)}$ is the Green function for $U=0$. Due to the appropriate scaling of $t_{i j}$ the self-energy becomes local in the limit $Z \rightarrow \infty$

$$
(\Sigma)_{i j, \sigma, n}=\Sigma_{i \sigma n} \delta_{i j}
$$

and its skeleton expansion depends only on the local Green function

$$
G_{i \sigma n}=(\boldsymbol{G})_{i i, \sigma, n}=\left\{\left[\left(\boldsymbol{G}^{(0)}\right)^{-1}-\boldsymbol{\Sigma}\right]^{-1}\right\}_{i i, \sigma, n} .
$$

Therefore $G_{i \sigma n}$ and $\Sigma_{i \sigma n}$ can also be calculated from an auxiliary impurity problem ${ }^{25}$ with the action

$$
\begin{aligned}
\mathcal{A}_{i}= & \sum_{n, \sigma} c_{i \sigma}^{*}\left(i \omega_{n}\right) \mathcal{G}_{i \sigma n}^{-1} c_{i \sigma}\left(i \omega_{n}\right) \\
& -U \int_{0}^{\beta} c_{i \uparrow}^{*}(\tau) c_{i \uparrow}(\tau) c_{i \downarrow}^{*}(\tau) c_{i \downarrow}(\tau) d \tau,
\end{aligned}
$$

according to

$$
\begin{aligned}
G_{i \sigma n} & =\left\langle c_{i \sigma}^{*}\left(i \omega_{n}\right) c_{i \sigma}\left(i \omega_{n}\right)\right\rangle_{\mathcal{A}_{i}} \\
& =\left[\mathcal{G}_{i \sigma n}^{-1}-\Sigma_{i \sigma n}\right]^{-1},
\end{aligned}
$$

so that the so-called Weiss field $\mathcal{G}_{i \sigma n}$ can be eliminated and two equations, (53) and (55) remain for $G_{i \sigma n}$ and $\Sigma_{i \sigma n}$.

The DMFT equations (53)-(56) are thus a closed set of equations for the self-energy of the Hubbard model (48), and become exact in the limit $Z \rightarrow \infty$. In practice these equations are solved by iteration, and the dynamic impurity problem
(54)-(56) is solved approximately by numerical or diagrammatic methods. In addition one must evaluate the selfconsistency equation (53), i.e., $G_{i \sigma n}$ must be expressed in terms of $\Sigma_{i \sigma n}$ and the noninteracting spectrum, preferably involving only the DOS. It is also useful to obtain $\mathcal{G}_{i \sigma n}$ in terms of $G_{i \sigma n}$ for use in Eq. (54). Below these evaluations are performed for $t_{1}-t_{2}$ hopping on the Bethe lattice with $Z \rightarrow \infty$ for homogeneous phases and phases with broken sublattice symmetry. For homogeneous phases $\Sigma_{i \sigma n}$ and $G_{i \sigma n}$ are independent of $i$, whereas for broken sublattice symmetry on a bipartite lattice one has $\Sigma_{i \sigma n}=\Sigma_{\gamma \sigma n}$ and $G_{i \sigma n}=G_{\gamma \sigma n}$ where $\gamma=(-1)^{i}= \pm 1=A, B$ depending on the sublattice. We add homogeneous and staggered magnetic fields to the Hamiltonian,

$$
H_{\mathrm{ext}}=-\sum_{i \sigma}\left[h_{f}+h_{a f}(-1)^{i}\right] \sigma n_{i \sigma},
$$

i.e., with a local field $h_{i \sigma}=\left[h_{f}+(-1)^{i} h_{a f}\right] \sigma$, which allows one to detect ferromagnetic or antiferromagnetic phases.

\section{B. Self-consistency equations}

We now evaluate the self-consistency equation (53) for arbitrary (scaled) hopping $t_{i j}=t_{d_{i j}}^{*} / K^{d_{i j} / 2}$ on the Bethe lattice. The effective dispersion is then $\epsilon(\lambda)=\mathcal{F}(\lambda)$ with DOS $\rho(\epsilon)$, see Eqs. (19)-(22). In particular we consider $t_{1}-t_{2}$ hopping in the limit $Z \rightarrow \infty$ with DOS (28).

\section{Homogeneous phases}

In the homogeneous case all sites are equivalent, as described above. We thus set $h_{a f}=0$ but keep $h_{f}$, which possibly leads to a ferromagnetic response. The self-consistency equation (53) becomes

$$
\begin{gathered}
G_{\sigma n}=\int \frac{\rho(\epsilon)}{z_{\sigma n}-\epsilon} d \epsilon \\
=\int_{-2}^{2} \frac{\rho_{1}(\lambda)}{z_{\sigma n}-\mathcal{F}(\lambda)} d \lambda,
\end{gathered}
$$

where $z_{\sigma n}=i \omega_{n}+\mu+\sigma h_{f}-\Sigma_{n \sigma}, \mu$ is the chemical potential, and the dispersion relation $\mathcal{F}(\lambda)$ [see Eqs. (19) and (20)] has been substituted. The general result (59) thus yields the DMFT self-consistency equation in the homogeneous case for arbitrary hopping. The special case of $t_{1}-t_{2}$ hopping is discussed in Sec. V C.

\section{Phases with broken sublattice symmetry}

In the case of broken sublattice symmetry the local Green function and self-energy depend on $i$ through the sublattice index $\gamma$. Spontaneous breaking of this symmetry can be detected through the staggered magnetic field $h_{a f}$; We also keep the homogeneous field $h_{f}$ which permits phases with $\Sigma_{A \sigma n} \neq \Sigma_{B \bar{\sigma} n}$.

We start from the eigenbasis of the NN hopping Hamiltonian $\widetilde{H}_{1}$, i.e., $\widetilde{H}_{1}|\theta\rangle=\lambda(\theta)|\theta\rangle$; these eigenstates may later be identified with those of Ref. 23. We use the sublattice trans- 
formation $|\bar{\theta}\rangle=\Sigma_{i}(-1)^{i}|i\rangle\langle i \mid \theta\rangle$, which yields $\widetilde{H}_{1}|\bar{\theta}\rangle=-\lambda(\theta)|\bar{\theta}\rangle$, and introduce wave functions that have nonzero amplitudes only on sublattice $\gamma$, i.e., $|\gamma \theta\rangle=(|\theta\rangle+\gamma|\bar{\theta}\rangle) / \sqrt{2}$. Using the corresponding fermion operators $c_{\gamma \theta \sigma}$ we now transform the tight-binding part $H_{\text {hop }}$ of the Hubbard model with effective dispersion $\mathcal{F}[\lambda(\theta)]$,

$$
\begin{aligned}
H_{\text {hop }}+H_{\text {ext }}= & \sum_{\theta \sigma}\left(c_{A \theta \sigma}^{\dagger} c_{B \theta \sigma}^{\dagger}\right) \\
& \lambda(\theta)>0 \\
& \times\left(\begin{array}{cc}
\mathcal{F}_{+}[\lambda(\theta)]-h_{A \sigma} & \mathcal{F}_{-}[\lambda(\theta)] \\
\mathcal{F}_{-}[\lambda(\theta)] & \mathcal{F}_{+}[\lambda(\theta)]-h_{B \sigma}
\end{array}\right)\left(\begin{array}{c}
c_{A \theta \sigma} \\
c_{B \theta \sigma}
\end{array}\right),
\end{aligned}
$$

where $h_{\gamma \sigma}=\left(h_{f}+\gamma h_{a f}\right) \sigma$ and $\mathcal{F}_{ \pm}(\lambda)=[\mathcal{F}(\lambda) \pm \mathcal{F}(-\lambda)] / 2$. Note that the reduced interval for $\lambda(\theta)$ is analogous to the halving of the magnetic Brillouin zone for regular crystal lattices.

For the evaluation of the self-consistency equation (53) we include the self-energy and perform the matrix inversion. This yields the interacting local Green function as a function of the local self-energy,

$$
\begin{aligned}
G_{\gamma \sigma n} & =\sum_{\substack{\theta \\
\lambda(\theta)>0}} \frac{|\langle i \mid \gamma \theta\rangle|^{2}\left\{z_{\bar{\gamma} \sigma n}-\mathcal{F}_{+}[\lambda(\theta)]\right\}}{\prod_{\gamma^{\prime}}\left\{z_{\gamma^{\prime} \sigma n}-\mathcal{F}_{+}[\lambda(\theta)]\right\}-\mathcal{F}_{-}[\lambda(\theta)]^{2}} \\
& =\int_{-2}^{2} \frac{\rho_{1}(\lambda)\left[z_{\bar{\gamma} \sigma n}-\mathcal{F}_{+}(\lambda)\right]}{\prod_{\gamma^{\prime}}\left[z_{\gamma^{\prime} \sigma n}-\mathcal{F}_{+}(\lambda)\right]-\mathcal{F}_{-}(\lambda)^{2}} d \lambda,
\end{aligned}
$$

where $i$ is any site belonging to sublattice $\gamma$ and $z_{\gamma \sigma n}$ $=i \omega_{n}+\mu+h_{\gamma \sigma}-\Sigma_{\gamma \sigma n}$. Equation (61) thus yields the DMFT self-consistency equations for arbitrary hopping.

The general expressions Eqs. (59) and (61) are the central results of our paper regarding DMFT. Note that their derivation involved no counting of lattice paths or other combinatorial efforts. Rather they were made possible by the dispersion relation $\mathcal{F}(\lambda)$ [Eqs. (19) and (20)] which is due to the operator identity (9).

\section{C. $t_{1}-t_{2}$ hopping}

We now specialize to $t_{1}-t_{2}$ hopping [with dispersion relation (24)] and the limit $Z \rightarrow \infty$. For the homogeneous case we find from (59) that $G_{\sigma n}=G_{t_{1}^{*}, t_{2}^{*}}^{\infty}\left(z_{\sigma n}\right)$, where the latter function was obtained in Sec. IV B [Eqs. (32)-(35)]. The resulting Weiss field is discussed below [Eq. (66)].

For broken sublattice symmetry we use Eq. (61) with $\mathcal{F}_{+}(\lambda)=\left(\lambda^{2}-1\right) t_{2}^{*}$ and $\mathcal{F}_{-}(\lambda)=\lambda t_{1}^{*}$. Performing the integral in Eq. (61) we find

$$
G_{\gamma \sigma n}=\frac{1}{2 t_{2}^{*}}+\sum_{i=1}^{2} \frac{(-1)^{i}\left[z_{\bar{\gamma} \sigma n}-\left(\lambda_{i}^{2}-1\right) t_{2}^{*}\right] \sqrt{\lambda_{i}-2} \sqrt{\lambda_{i}+2}}{2\left(\lambda_{2}^{2}-\lambda_{1}^{2}\right) \lambda_{i} t_{2}^{* 2}},
$$

where $\pm \lambda_{i}$ are the poles of the integrand, given by

$$
\lambda_{i}=\sqrt{\mathcal{A} \pm \sqrt{\mathcal{A}^{2}-\mathcal{B}}},
$$

$$
\begin{aligned}
& \mathcal{A}=1+\frac{t_{2}^{*}\left(z_{A \sigma n}+z_{B \sigma n}\right)+t_{1}^{* 2}}{2 t_{2}^{* 2}}, \\
& \mathcal{B}=\left(\frac{z_{A \sigma n}}{t_{2}^{*}}+1\right)\left(\frac{z_{B \sigma n}}{t_{2}^{*}}+1\right),
\end{aligned}
$$

and all square roots are given by their principal branches. A more compact expression results if one solves for $z_{\gamma \sigma n}$ in terms of Green functions, i.e.,

$$
z_{\gamma \sigma n}=-t_{2}^{*}+\frac{1}{G_{\gamma \sigma n}\left(1-t_{2}^{*} G_{\gamma \sigma n}\right)}+\frac{t_{1}^{* 2} G_{\bar{\gamma} \sigma n}\left(1-t_{2}^{*} G_{\bar{\gamma} \sigma n}\right)}{\left(1-t_{2}^{*} \sum_{\gamma^{\prime}} G_{\gamma^{\prime} \sigma n}\right)^{2}} .
$$

In the present notation the Weiss field is given by $\mathcal{G}_{\gamma \sigma n}^{-1}=i \omega_{n}+\mu+h_{\gamma \sigma}+G_{\gamma \sigma n}^{-1}-z_{\gamma \sigma n}$. From Eq. (64) follows the exact relation between Weiss field and interacting local Green function as

$$
\mathcal{G}_{\gamma \sigma n}^{-1}=i \omega_{n}+\mu+h_{\gamma \sigma}-\frac{t_{1}^{* 2} G_{\bar{\gamma} \sigma n}\left(1-t_{2}^{*} G_{\bar{\gamma} \sigma n}\right)}{\left(1-t_{2}^{*} \sum_{\gamma^{\prime}} G_{\gamma^{\prime} \sigma n}\right)^{2}}-\frac{t_{2}^{* 2} G_{\gamma \sigma n}}{\left(1-t_{2}^{*} G_{\gamma \sigma n}\right)} .
$$

Thus for nonrandom $t_{1}-t_{2}$ hopping the self-consistency equation is more complicated than for the two-sublattice fully frustrated model with random hopping, ${ }^{29,36}$ in which all terms in parentheses are absent. Our algebraic derivation validates Ref. 40, where Eq. (65) was obtained using RPE methods, which required the classification of many complicated hopping processes. We also note that for $U=0 \mathrm{Eq}$. (65) reduces to the same quartic equation that follows from Ref. 41 for the noninteracting Green function [Eq. (34)].

The corresponding equation for the homogeneous case is obtained by setting $h_{a f}$ to zero and dropping sublattice indices, i.e.,

$$
\mathcal{G}_{\sigma n}^{-1}=i \omega_{n}+\mu+\sigma h_{f}-\left(\frac{t_{1}^{* 2}\left(1-t_{2}^{*} G_{\sigma n}\right)}{\left(1-2 t_{2}^{*} G_{\sigma n}\right)^{2}}+\frac{t_{2}^{* 2}}{\left(1-t_{2}^{*} G_{\sigma n}\right)}\right) G_{\sigma n} .
$$

Returning to Eq. (65) we note that for pure NN hopping $\left(t_{2}^{*}=0\right)$ it reduces to the standard expression ${ }^{29}$

$$
\mathcal{G}_{\gamma \sigma n}^{-1}=i \omega_{n}+\mu+h_{\gamma \sigma}-t_{1}^{* 2} G_{\bar{\gamma} n \sigma},
$$

while for pure NNN hopping $\left(t_{1}^{*}=0\right)$

$$
\mathcal{G}_{\gamma \sigma n}^{-1}=i \omega_{n}+\mu+h_{\gamma \sigma}-\frac{t_{2}^{* 2} G_{\gamma \sigma n}}{1-t_{2}^{*} G_{\gamma \sigma n}} .
$$

In the former case there is strong sublattice mixing since hopping takes place only between different sublattices. In the latter case we find no mixing at all since hopping is allowed only between sites in the same sublattice.

\section{DISCUSSION}

On the Bethe lattice and the triangular Husimi cactus the number of possible paths joining two points is determined 
only by the topological distance between these points. Using this property we derived the operator relation (9) and obtained the spectrum of arbitrary tight-binding Hamiltonians, without the need for complicated geometrical constructions of other methods.

In Sec. IV the density of states was calculated analytically for several classes of tight-binding Hamiltonians on the Bethe lattice. As the NNN hopping amplitude increases it becomes asymmetric and develops a square-root singularity at a band edge. For the Hubbard model such a shape of the DOS is known ${ }^{51-53}$ to support metallic ferromagnetism away from half-filling, and to suppress antiferromagnetism near half-filling.

In Sec. IV D we showed that for any given DOS one can determine the corresponding hopping parameters on a Bethe lattice. This result is useful in particular in the context of dynamical mean-field theory. Namely, with this inverse construction it is now possible to mimic any kind of van-Hove singularity or other band structure even on the Bethe lattice.

Furthermore, we derived the exact self-consistency equations of dynamical mean-field theory in Sec. V for arbitrary hopping. In the particular case of $t_{1}-t_{2}$ hopping these equations differ from those used in previous investigations, ${ }^{29,36,38,39}$ which are therefore found to apply only to random hopping. The exact DMFT setup derived in this paper [DOS (28) and Weiss field (65)] can be expected to lead to new quantitative results for the phase diagram of the $t_{1}$ $-t_{2}$ Hubbard model on the Bethe lattice. This is clear from the exact relation (65) between the Weiss field and the interacting local Green function, since the Green function now appears also in the denominator, thus leading to resonances in the Weiss field. It will be most interesting to study the possible solutions obtained within this framework in detail.

In conclusion, the method and results presented in this paper lay the foundation for systematic studies of correlated electronic systems on a Bethe lattice. Possible future applications include geometrical frustration in itinerant systems or quantum magnets, and weak-localization effects in disordered systems. Indeed, the relation (9) may also become quite useful for future investigations in statistical mechanics.

\section{ACKNOWLEDGMENTS}

The authors thank N. Blümer, R. Bulla, G. Kotliar, and W. Metzner for useful discussions. This work was supported in part by the SFB 484 of the Deutsche Forschungsgemeinschaft (DFG). Financial support of one of the authors (K.B.) through KBN-2 P03B 08224 is also acknowledged.

\section{APPENDIX: THE TRIANGULAR HUSIMI CACTUS}

The triangular Husimi cactus is a set of triangles connected in such a way that each vertex belongs to $Z$ triangles and each edge belongs to only one triangle (see Fig. 9). Below we show how to express powers of the NN hopping Hamiltonian $H_{1}$ in terms of the hopping Hamiltonian $H_{d}$ between sites with topological distance $d$ [see Eq. (1b)]. For brevity we again set $K=Z-1$ and $p=Z / K$, and employ scaled operators $\widetilde{H}_{d}=H_{d} / K^{d / 2}$.

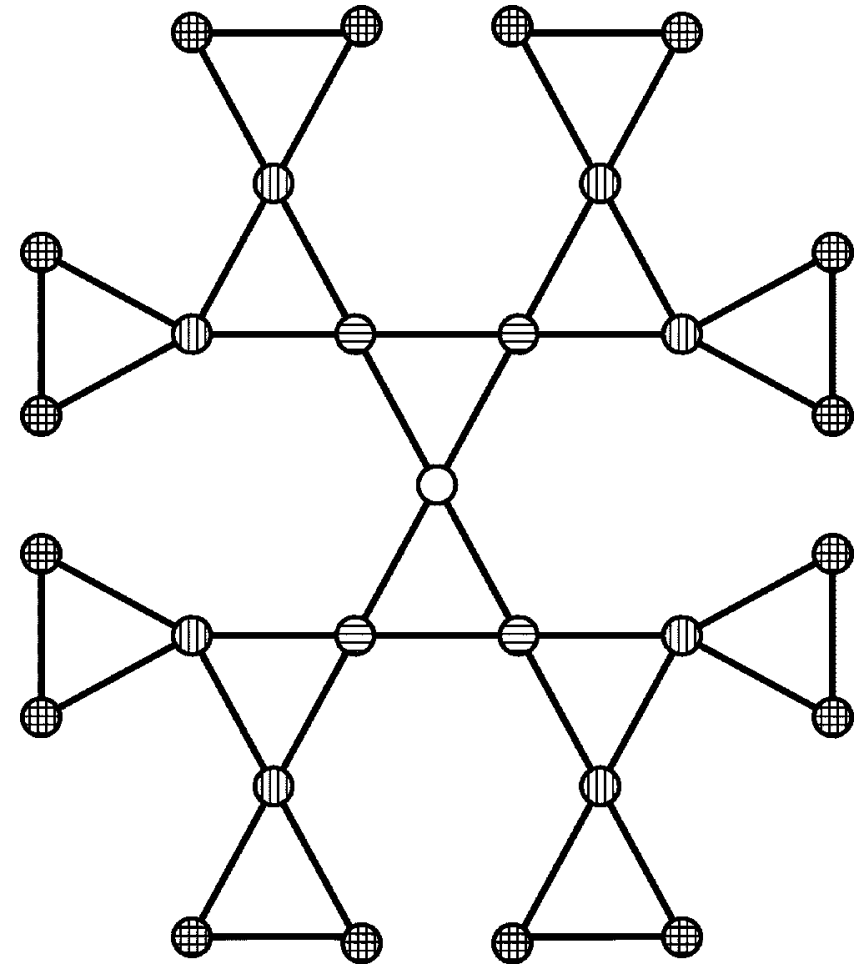

FIG. 9. Part of the triangular Husimi cactus with $Z$ triangles connected to each site; here $Z=2$. Any two sites are connected by a unique shortest path of bonds. The lattice is infinite and all sites are equivalent. The shading of sites has the same meaning as in Fig. 1.

One can check directly that the Husimi cactus is distance regular, i.e., the number of paths between two vertices $i$ and $j$ depends only on their topological distance. This shows that the absence of closed loops as on the Bethe lattice is not a necessary condition for this property. The number $a_{n}^{(d)}$ of paths of length $n$ between two points with distance $d_{i j}=d$ satisfies recursion relations very similar to those on the Bethe lattice, for $n, d \geqslant 0$ we find $a_{n+1}^{(d+1)}=2 K a_{n}^{(d+2)}+a_{n}^{(d+1)}+a_{n}^{(d)}$, $a_{n+1}^{(0)}=2 Z a_{n}^{(1)}, a_{0}^{(d)}=\delta_{0 d}$, and $a_{n}^{(n)}=1$. Using Eq. (4) we thus find for the first few powers of $H_{1}$,

$$
\begin{gathered}
\left(H_{1}\right)^{2}=2 Z \perp+H_{1}+H_{2}, \\
\left(H_{1}\right)^{3}=2 Z \perp+(4 Z-1) H_{1}+2 H_{2}+H_{3}, \\
\left(H_{1}\right)^{4}=(4 Z-1) 2 Z \perp+5(2 Z-1) H_{1}+(6 Z-1) H_{2}+3 H_{3}+H_{4} .
\end{gathered}
$$

Note that due to the presence of closed loops there are also paths of odd length joining vertices with even distance and vice versa.

We now proceed similar to Sec. III. The recursion relations for the generating function $F_{d}(u)=\sum_{n=0}^{\infty} a_{n}^{(d)} u^{n}$ can again be solved by an ansatz ${ }^{46} F_{d}(u)=f(u)[u g(u)]^{d}$. After some algebra we obtain the operator identity 


$$
\frac{(1+2 x)(1-x)}{1-x\left(H_{1}-1\right)+2 K x^{2}}=\sum_{d=0}^{\infty} H_{d} x^{d}
$$

relating nearest-neighbor and long-range hopping Hamiltonians on the Husimi cactus. (We omit explicit expressions for the coefficients $a_{n}^{(d)}$ or $A_{n}^{(d)}$.) Note that Eq. (A2) is somewhat more complicated than the corresponding relation for the $\mathrm{Be}$ the lattice [Eq. (9)].

The Green function (13) can now be obtained directly from Eq. (A2), similar to Sec. III B. We find

$$
G_{i j}(z)=\left\langle i\left|\left(z-t_{1} H_{1}\right)^{-1}\right| j\right\rangle=\frac{x^{d_{i j}}}{z-2 x Z t_{1}},
$$

where $x=\left[z / t_{1}-1+\sqrt{\left(z / t_{1}-1\right)^{2}-8 K}\right] /(4 K)$ and $d_{i j}$ again denotes the topological distance between sites $i$ and $j$. For $\widetilde{H}_{1}$ (i.e., $t_{1}=1 / \sqrt{K}$ ) the DOS is given by

$$
\rho_{1}(\lambda)=\delta_{K 1} \frac{\delta(\lambda+2)}{3}+\frac{p}{2 \pi} \frac{\sqrt{8-(\lambda-1 / \sqrt{K})^{2}}}{(p+\lambda / \sqrt{K})(2 p-\lambda / \sqrt{K})},
$$

implying a continuous spectrum in the interval $1 / \sqrt{K}-\sqrt{8}$ $\leqslant \lambda \leqslant 1 / \sqrt{K}+\sqrt{8}$ with an additional delta peak in the case $K=1$. Previous derivations of (A4) can be found for $K=1$ in Ref. 22 and for $K>1$ in Ref. 54. Note that Eq. (A4) reduces to a semielliptical DOS for $Z \rightarrow \infty$.
As an application we now calculate the DOS for the Hamiltonian with $t_{1}-t_{2}$ hopping, i.e.,

$$
H_{t_{1}, t_{2}^{*}}^{*}=t H_{1}+t_{2} H_{2}=t_{1}^{*} \tilde{H}_{1}+t_{2}^{*} \widetilde{H}_{2}=t H_{1}+t_{2}\left(H_{1}^{2}-H_{1}-2 Z \perp\right),
$$

due to Eq. (A1a). As before we use the scaling $t_{1}=t_{1}^{*} / \sqrt{K}$ and $t_{2}=t_{2}^{*} / K$ and find the eigenvalues $\epsilon$ of $H$ in terms of the eigenvalues $\lambda$ of $\tilde{H}_{1}$, i.e.,

$$
\epsilon(\lambda)=t_{2}^{*} \lambda^{2}+\left(t_{1}^{*}-t_{2}^{*} / \sqrt{K}\right) \lambda-2 p t_{2}^{*},
$$

which has the roots $\lambda_{1,2}(\epsilon)=\left(\xi \pm \sqrt{\xi^{2}+\eta}\right) /\left(2 t_{2}^{*}\right)$, where

$$
\xi=\frac{t_{2}^{*}}{\sqrt{K}}-t_{1}^{*}, \quad \eta=4 t_{2}^{*}\left(2 p t_{2}^{*}+\epsilon\right) .
$$

Finally the DOS is obtained by changing variables in Eq. (A4),

$$
\rho_{t_{1}, t_{2}^{*}}^{*}(\epsilon)=\delta_{K 1} \frac{\delta\left[\epsilon+2\left(t_{1}^{*}-t_{2}^{*}\right)\right]}{3}+\sum_{i=1}^{2} \frac{\rho_{1}\left[\lambda_{i}(\epsilon)\right]}{\sqrt{\xi^{2}+\eta}} .
$$

The Husimi cactus is not a bipartite lattice, and the symmetry $\rho_{t_{1}, t_{2}^{*}}{ }^{*}(\epsilon)=\rho_{-t_{1}}{ }^{*}, t_{2}^{*}(\epsilon)$ holds only for $K=\infty$; this can be seen from Eq. (A6). We omit plots of (A8) since its behavior with varying $t_{2}^{*}$ is rather similar to the DOS (26) for the Bethe lattice.
${ }^{1}$ H. A. Bethe, Proc. R. Soc. London, Ser. A 150, 552 (1935).

${ }^{2}$ R. J. Baxter, Exactly Solved Models in Statistical Mechanics (Academic, London, 1982).

${ }^{3}$ T. P. Eggarter, Phys. Rev. B 9, 2989 (1974).

${ }^{4}$ E. Müller-Hartmann and J. Zittartz, Phys. Rev. Lett. 33, 893 (1974).

${ }^{5}$ F. Yndurain, Phys. Lett. 62, 93 (1977); F. Yndurain, R. Barrio, R. J. Elliott, and M. F. Thorpe, Phys. Rev. B 28, 3576 (1983).

${ }^{6}$ C.-K. Hu and N. Sh. Izmailian, Phys. Rev. E 58, 1644 (1998), and references therein.

${ }^{7}$ M. E. Fisher and J. W. Essam, J. Math. Phys. 2, 609 (1964)

${ }^{8}$ J. Chalupa, P. L. Leath, and G. R. Reich, J. Phys. C 12, L31 (1979).

${ }^{9}$ Z. Q. Zhang, T. C. Li, and F. C. Pu, J. Phys. A 16, 2267 (1983).

${ }^{10}$ R. Abou-Chacra, D. J. Thouless, and P. W. Anderson, J. Phys. C 6, 1734 (1973); R. Abou-Chacra, and D. J. Thouless, ibid. 7, 65 (1974).

${ }^{11}$ A. D. Mirlin and Y. V. Fyodorov, Nucl. Phys. B 366, 507 (1991); Phys. Rev. Lett. 72, 526 (1994); J. Phys. (France) 4, 655 (1994).

${ }^{12}$ K. Efetov, Supersymmetry in Disorder and Chaos (Cambridge University Press, Cambridge, U.K., 1997).

${ }^{13}$ M. R. Zirnbauer, Phys. Rev. B 34, 6394 (1986).

${ }^{14}$ P. D. Gujrati, Phys. Rev. Lett. 74, 809 (1995).

${ }^{15}$ D. Weaire and M. F. Thorpe, Phys. Rev. B 4, 2508 (1971); M. F. Thorpe and D. Weaire, ibid. 4, 3518 (1971); M. F. Thorpe, D. Weaire, and R. Alben, ibid. 7, 3777 (1973).

${ }^{16}$ J. A. Verges, Phys. Rev. Lett. 53, 2270 (1984).
${ }^{17}$ R. B. Laughlin and J. D. Joannopoulos, Phys. Rev. B 16, 2942 (1977).

${ }^{18}$ N. Mingo and L. Yang, Phys. Rev. B 68, 245406 (2003).

${ }^{19}$ W. F. Brinkman and T. M. Rice, Phys. Rev. B 2, 1324 (1970).

${ }^{20}$ M. Chen, L. Onsager, J. Bonner, and J. Nagle, J. Chem. Phys. 60, 405 (1974).

${ }^{21}$ E. N. Economou, Green's Functions in Quantum Physics (Springer-Verlag, Berlin, 1979).

${ }^{22}$ M. F. Thorpe, in Excitations in Disordered Systems, edited by M. F. Thorpe (Plenum, New York, 1981), pp. 85-107.

${ }^{23}$ G. D. Mahan, Phys. Rev. B 63, 155110 (2001). Note that in Ref. 23 only "shell-symmetric" eigenstates are obtained; only these states contribute to the Green function and the density of states for an infinite Bethe lattice.

${ }^{24}$ W. Metzner and D. Vollhardt, Phys. Rev. Lett. 62, 324 (1989).

${ }^{25}$ A. Georges and G. Kotliar, Phys. Rev. B 45, 6479 (1992).

${ }^{26}$ M. Jarrell, Phys. Rev. Lett. 69, 168 (1992).

${ }^{27}$ D. Vollhardt, in Correlated Electron Systems, edited by V. J. Emery (World Scientific, Singapore, 1993), p. 57.

${ }^{28}$ Th. Pruschke, M. Jarrell, and J. K. Freericks, Adv. Phys. 44, 187 (1995).

${ }^{29}$ For a comprehensive review see A. Georges, G. Kotliar, W. Krauth, and M. J. Rozenberg, Rev. Mod. Phys. 68, 13 (1996).

${ }^{30}$ G. Kotliar and D. Vollhardt, Phys. Today 57, 53 (2004).

${ }^{31}$ R. Bulla, Phys. Rev. Lett. 83, 136 (1999).

${ }^{32}$ M. J. Rozenberg, R. Chitra, and G. Kotliar, Phys. Rev. Lett. 83, 3498 (1999).

${ }^{33}$ J. Joo and V. Oudovenko, Phys. Rev. B 64, 193102 (2001). 
${ }^{34}$ R. Bulla, T. A. Costi, and D. Vollhardt, Phys. Rev. B 64, 045103 (2001).

${ }^{35}$ N. Blümer, Ph. D. thesis, Universität Augsburg, 2002; available from http://www.physik.uni-augsburg.de/theo3/ diss.vollha.en.shtml; N. Blümer and E. Kalinowski, Phys. Rev. B 71, 195102 (2005).

${ }^{36}$ M. J. Rozenberg, X. Y. Zhang, and G. Kotliar, Phys. Rev. Lett. 69, 1236 (1992); M. J. Rozenberg, G. Kotliar, and X. Y. Zhang, Phys. Rev. B 49, 10181 (1994).

${ }^{37}$ D. B. McWhan and J. P. Remeika, Phys. Rev. B 2, 3734 (1970); D. B. McWhan, A. Menth, J. P. Remeika, W. F. Brinkman, and T. M. Rice, ibid. 7, 1920 (1973).

${ }^{38}$ M. J. Rozenberg, G. Kotliar, H. Kajueter, G. A. Thomas, D. H. Rapkine, J. M. Honig, and P. Metcalf, Phys. Rev. Lett. 75, 105 (1995).

${ }^{39}$ R. Zitzler, N.-H. Tong, Th. Pruschke, and R. Bulla, Phys. Rev. Lett. 93, 016406 (2004).

${ }^{40}$ M. H. Radke de Cuba, Ph. D. thesis (RWTH Aachen, 2002), available from http://www.bth.rwth-aachen.de/job/disslist.pl

${ }^{41}$ D. Tanasković, V. Dobrosavljević, and G. Kotliar (private communication).

${ }^{42}$ M. Kollar M. Eckstein, K. Byczuk, N. Blümer, P. van Dongen, M. H. Radke de Cuba, W. Metzner, D. Tanasković, V. Dobrosavljević, G. Kotliar, and D. Vollhardt, cond-mat/0504637.

${ }^{43}$ J. A. de Miranda-Neto and F. Moraes, J. Phys. I 2, 1657 (1992); 3, 29 (1993). This embedding is not unique, see, e.g., B. Söderberg, Phys. Rev. E 47, 4582 (1993).
${ }^{44}$ Selected Topics in Graph Theory, edited by L. W. Beineke and R. J. Wilson (Academic, New York, 1978), Graph Theory and Theoretical Physics, edited by F. Harry (Academic, New York, 1967).

${ }^{45}$ M. A. Fiol, Discrete Math. 246, 111 (2002).

${ }^{46}$ The recursion relations for $a_{n}^{(d)}$ are those of a so-called Riordan array, which has precisely this type of generating function; see, e.g., D. Merlini, D. G. Rogers, R. Sprugnoli, and M. C. Verri, Can. J. Math. 49, 301 (1997).

${ }^{47}$ Pocketbook of Mathematical Functions, edited by M. Abramowitz and I. A. Stegun (Harry Deutsch, Thun, 1984).

${ }^{48}$ P. van Dongen and D. Vollhardt, Phys. Rev. B 40, 7252 (1989).

${ }^{49}$ N. Blümer and P. G. J. van Dongen, in Concepts in Electron Correlation, edited by A. C. Hewson and V. Zlatic, NATO Science Series (Kluwer, Dordrecht, 2003).

${ }^{50}$ M. Kollar, Int. J. Mod. Phys. B 16, 3491 (2002).

${ }^{51}$ J. Wahle, N. Blümer, J. Schlipf, K. Held, and D. Vollhardt, Phys. Rev. B 58, 12749 (1998).

${ }^{52}$ S. Daul and R. M. Noack, Z. Phys. B: Condens. Matter 103, 293 (1997); Phys. Rev. B 58, 2635 (1998).

${ }^{53}$ D. Vollhardt, N. Blümer, K. Held, and M. Kollar, in BandFerromagnetism: Ground-State and Finite-Temperature Phenomena, edited by K. Baberschke, M. Donath, and W. Nolting, Lect. Notes Phys. Vol. 580 (Springer, Heidelberg, 2001), p. 191.

${ }^{54}$ J. Wahle, Diploma thesis, Universität Augsburg, 1997), available from http://www.physik.uni-augsburg.de/theo3/ diplom.vollha.en.shtml 